\title{
Correction to: Outcome of kidney function after ischaemic and zero-ischaemic laparoscopic and open nephron-sparing surgery for renal cell cancer
}

Jan Ebbing ${ }^{1,6^{*}}$, Felix Menzel ${ }^{2}$, Paolo Frumento ${ }^{3}$, Kurt Miller ${ }^{2}$ Bernhard Ralla², Tom Florian Fuller ${ }^{2}$, Jonas Busch², Justin William Collins ${ }^{4}$, Christofer Adding ${ }^{4}$, Hans Helge Seifert ${ }^{1}$, Peter Ardelt ${ }^{1}$, Christian Wetterauer ${ }^{1}$, Timm Westhoff ${ }^{5}$ and Carsten Kempkensteffen ${ }^{2,7}$

Correction to: BMC Nephrology (2019) 20:40 https://doi.org/10.1186/s12882-019-1215-3

Following publication of the original article [1], it was reported that Fig. 1i and Fig. $1 \mathrm{j}$ were omitted due to a typesetting mistake. In this Correction, the complete Fig. 1 is shown and the original publication of this article has been updated to correct this. The publisher apologises to the authors and readers for the inconvenience.

Additionally, the authors reported that the caption of Table 4 was incorrectly presented as "Multiple linear regression analysis". The correct presentation of this table caption is "Multiple regression analysis". And Table 4 with its corrected caption can be found on page 3-4.

\begin{abstract}
Author details
${ }^{1}$ University Hospital Basel, Urological University Clinic Basel-Liestal, Spitalstrasse 21, 4051 Basel, Switzerland. ²Department of Urology, Charité University Hospital, Berlin, Germany. ${ }^{3}$ Karolinska Institutet, Unit of Biostatistics, Institute of Environmental Medicine (IMM), Stockholm, Sweden. ${ }^{4}$ Department of Molecular Medicine and Surgery (MMK), Karolinska Institutet, Stockholm, Sweden. ${ }^{5}$ Marien Hospital Herne - University Clinic of the Ruhr-University Bochum, Medical Clinic I, Herne, Germany. ${ }^{6}$ Department of Urology, Karolinska - University Hospital, Solna, Stockholm, Sweden. 'Department of Urology, Franziskus Hospital Berlin, Berlin, Germany.
\end{abstract}

Received: 7 February 2019 Accepted: 7 February 2019

Published online: 07 March 2019

\section{Reference}

1. Ebbing, et al. Outcome of kidney function after ischaemic and zeroischaemic laparoscopic and open nephron-sparing surgery for renal cell cancer. BMC Nephrol. 2019;20:40 https://doi.org/10.1186/s12882-019-1215-3.

\footnotetext{
* Correspondence: jan.ebbing@usb.ch

1 University Hospital Basel, Urological University Clinic Basel-Liestal,

Spitalstrasse 21, 4051 Basel, Switzerland

${ }^{6}$ Department of Urology, Karolinska - University Hospital, Solna, Stockholm,

Sweden

Full list of author information is available at the end of the article
}

(c) The Author(s). 2019 Open Access This article is distributed under the terms of the Creative Commons Attribution 4.0 International License (http://creativecommons.org/licenses/by/4.0/), which permits unrestricted use, distribution, and reproduction in any medium, provided you give appropriate credit to the original author(s) and the source, provide a link to the Creative Commons license, and indicate if changes were made. The Creative Commons Public Domain Dedication waiver (http://creativecommons.org/publicdomain/zero/1.0/) applies to the data made available in this article, unless otherwise stated. 


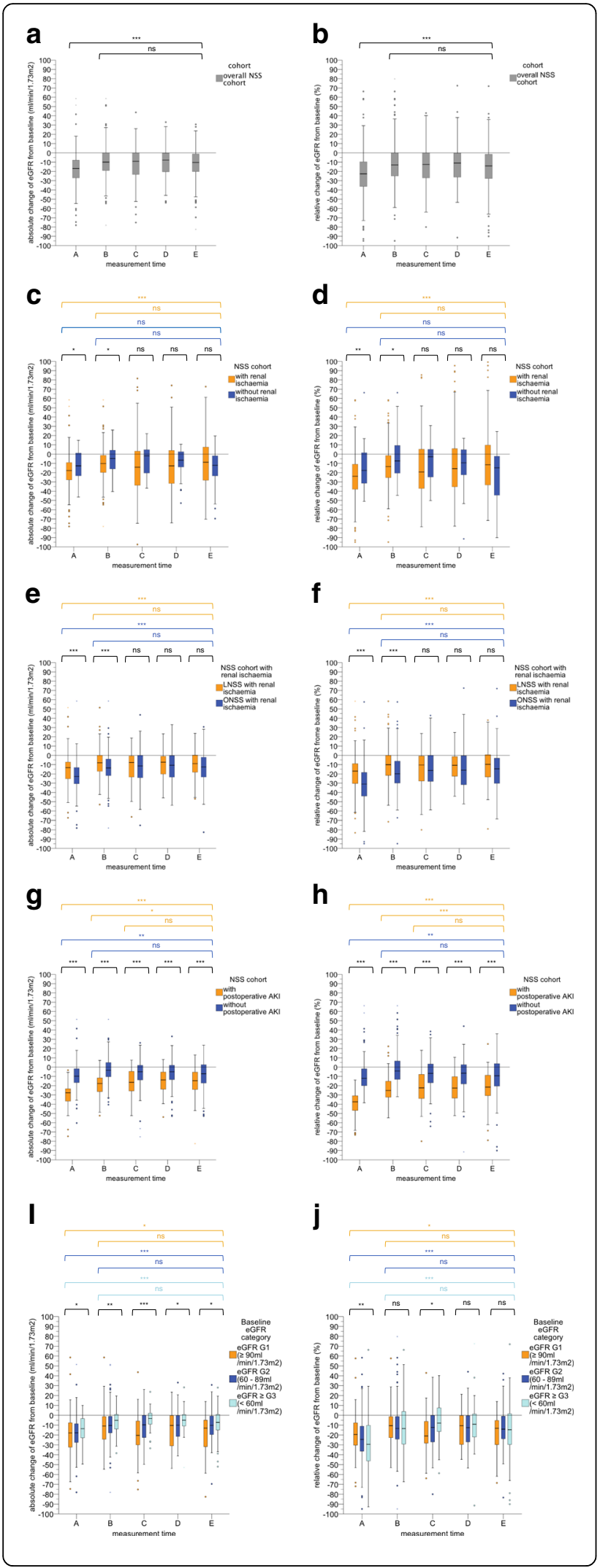

Fig. 1 Box plots showing the postoperative course of the absolute $(\mathbf{a} / \mathbf{c} / \mathbf{e} / \mathbf{g} / \mathbf{i})$ and relative $(\mathbf{b} / \mathbf{d} / \mathbf{f} / \mathbf{h} / \mathbf{j})$ change (\%) in eGFR at measurement times A-E for $(\mathbf{a} / \mathbf{b})$ the overall NSS cohort (NSS-C), (c/ d) the NSS group with intraoperative renal ischaemia (NSS-RI) and without intraoperative renal ischaemia (NSS-NRI), (e/f) the LNSS group with intraoperative renal ischaemia (LNSS-RI), the ONSS group with intraoperative renal ischaemia (ONSS-RI), $(\mathbf{g} / \mathbf{h})$ the NSS group with postoperative AKI (NSS-AKI), the NSS group without postoperative AKI (NSS-NAKI), and (i//j) NSS group with a baseline eGFR category G1 (NSS-G1), NSS group with a baseline eGFR category G2 (NSS-G2), and NSS group with a baseline eGFR category $\geq G 3$ (NSS $\geq$ G3). Definition of measurement times a-e: (a) highest change in eGFR from baseline during the planned hospital stay at a median of 1 day postoperatively (IQR, 1-2), (b) change in eGFR from baseline prior to discharge from hospital at a median of 4 days postoperatively (IQR, 2-6), (c) change in eGFR from baseline at a median of 47 days postoperatively (IQR, 30-105), (d) a median of 13 months postoperatively (IQR, 12-15), and (e) a median of 50 months postoperatively (IQR, 35-81). Asterisks indicate significant changes from baseline in the level of absolute and relative changes in eGFR over the course of the observation period (Friedman's test as a post hoc pairwise multiple comparison test) or between the compared groups at each measurement time (non-parametric Mann-Whitney $U$ test). ${ }^{*} p<0.05,{ }^{* *} p<0.01,{ }^{* * *} p<0.001$, (ns) not significant. eGFR, estimated glomerular filtration rate; NSS, nephron-sparing surgery; LNSS, laparoscopic nephron-sparing surgery; ONSS, open nephronsparing surgery; AKI, acute kidney injury; $\mathrm{IQR}$, interquartile range 
Table 4 Multiple regression analysis

\begin{tabular}{|c|c|c|c|}
\hline $\mathrm{a}$ (model 1) & $\begin{array}{l}\text { Regression } \\
\text { coefficient } \\
\beta\end{array}$ & $\begin{array}{l}\text { Multiple linear regression } \\
95 \% \mathrm{Cl}\end{array}$ & $p$-value \\
\hline Baseline eGFR (mL/ml/1.73 m²) & -0.20 & $-0.38--0.02$ & 0.03 \\
\hline Baseline Haemoglobin (mg/dL) & 0.51 & $-0.86-1.89$ & 0.46 \\
\hline Tumour diameter $(\mathrm{cm})$ & 0.67 & $-0.43-1.76$ & 0.24 \\
\hline Tumour locus central (ref.) vs. peripheral & 0.43 & $-3.80-4.67$ & 0.84 \\
\hline Surgical approach LNSS (ref.) vs. ONSS & -13.48 & $-17.65--9.32$ & $<0.001$ \\
\hline Sex male (ref.) vs. female & -3.28 & $-7.80-1.25$ & 0.16 \\
\hline Age (years) & -0.17 & $-0.36-0.01$ & 0.06 \\
\hline $\mathrm{BMI}\left(\mathrm{kg} / \mathrm{m}^{2}\right)$ & -0.88 & $-1.36--0.41$ & $<0.001$ \\
\hline Hypertension no (ref.) vs. yes & -0.78 & $-4.75-3.18$ & 0.70 \\
\hline Ischaemia time (min) & -0.27 & $-0.41--0.13$ & $<0.001$ \\
\hline Operative time (min) & -0.06 & $-0.09--0.03$ & $<0.001$ \\
\hline Preoperative ureter stenting no (ref.) vs. yes & -0.46 & $-5.64-4.71$ & 0.86 \\
\hline Intraoperative blood transfusions no (ref.) vs. yes & -3.29 & $-11.91-5.33$ & 0.45 \\
\hline Postoperative complications no (ref.) vs. yes & -3.36 & $-8.42-1.70$ & 0.19 \\
\hline Clavien-Dindo score $<3$ (ref.) vs. $\geq 3$ & -10.98 & $-18.47--3.48$ & 0.004 \\
\hline b (model 2) & $\begin{array}{l}\text { Regression } \\
\text { coefficient } \\
\beta\end{array}$ & $\begin{array}{l}\text { Multiple linear regression } \\
95 \% \mathrm{Cl}\end{array}$ & $p$-value \\
\hline Baseline eGFR (mL/ml/1.73 m²) & -0.29 & $-0.49--0.09$ & 0.005 \\
\hline Baseline Haemoglobin (mg/dL) & -0.32 & $-1.95-1.31$ & 0.70 \\
\hline Relative change of eGFR from baseline at time A (\%) & 0.18 & $0.03-0.33$ & 0.02 \\
\hline AKI 48 h p.o. no (ref.) vs. yes & -2.11 & $-9.01-4.79$ & 0.55 \\
\hline Tumour diameter $(\mathrm{cm})$ & -1.76 & $-2.87--0.66$ & 0.002 \\
\hline Tumour locus central (ref.) vs. peripheral & -0.30 & $-5.14-4.54$ & 0.90 \\
\hline Surgical approach LNSS (ref.) vs. ONSS & 1.13 & $-4.17--6.44$ & 0.67 \\
\hline Sex male (ref.) vs. female & 1.63 & $-3.43-6.70$ & 0.53 \\
\hline Age (years) & -0.10 & $-0.33-0.13$ & 0.40 \\
\hline $\mathrm{BMI}(\mathrm{kg} / \mathrm{m} 2)$ & 0.15 & $-0.39-0.70$ & 0.58 \\
\hline Hypertension no (ref.) vs. yes & -2.11 & $-6.82-2.60$ & 0.38 \\
\hline Ischaemia time (min) & 0.03 & $-0.14-0.21$ & 0.72 \\
\hline Operative time (min) & 0.01 & $-0.03-0.05$ & 0.54 \\
\hline Preoperative ureter stenting no (ref.) vs. yes & -4.35 & $-10.46-1.77$ & 0.16 \\
\hline Intraoperative blood transfusions no (ref.) vs. yes & 4.12 & $-6.29-14.52$ & 0.44 \\
\hline Postoperative complications no (ref.) vs. yes & 1.20 & $-4.77-7.18$ & 0.69 \\
\hline Clavien-Dindo score $<3$ (ref.) vs. $\geq 3$ & 4.43 & $-4.28-13.14$ & 0.32 \\
\hline c (model 3) & OR & $\begin{array}{l}\text { Multiple logistic regression } \\
95 \% \mathrm{Cl}\end{array}$ & $p$-value \\
\hline Baseline eGFR (mL/ml/1.73 m²2) & 0.99 & $0.96-1.01$ & 0.30 \\
\hline Baseline Haemoglobin (mg/dl) & 0.85 & $0.70-1.03$ & 0.10 \\
\hline Tumour diameter $(\mathrm{cm})$ & 0.94 & $0.81-1.08$ & 0.35 \\
\hline Tumour locus central (ref.) vs. peripheral & 1.20 & $0.70-2.05$ & 0.51 \\
\hline Surgical approach LNSS (ref.) vs. ONSS & 3.87 & $2.17-6.92$ & $<0.001$ \\
\hline Sex male (ref.) vs. female & 2.51 & $1.35-4.67$ & 0.004 \\
\hline Age (years) & 1.01 & $0.99-1.04$ & 0.26 \\
\hline
\end{tabular}


Table 4 Multiple regression analysis (Continued)

\begin{tabular}{|c|c|c|c|}
\hline$\overline{\mathrm{BMI}}\left(\mathrm{kg} / \mathrm{m}^{2}\right)$ & 1.13 & $1.06-1.21$ & $<0.001$ \\
\hline Hypertension no (ref.) vs. yes & 1.05 & $0.63-1.74$ & 0.85 \\
\hline Ischaemia time (min) & 1.02 & $1.00-1.04$ & 0.046 \\
\hline Operative time (min) & 1.01 & $1.00-1.01$ & 0.002 \\
\hline Preoperative ureter stenting no (ref.) vs. yes & 0.92 & $0.46-1.83$ & 0.81 \\
\hline Intraoperative blood transfusions no (ref.) vs. yes & 0.73 & $0.22-2.45$ & 0.61 \\
\hline Postoperative complications no (ref.) vs. yes & 1.79 & $0.92-3.48$ & 0.08 \\
\hline Clavien-Dindo score $<3$ (ref.) vs. $\geq 3$ & 2.14 & $0.68-6.72$ & 0.19 \\
\hline $\mathrm{d}$ (model 4) & OR & $\begin{array}{l}\text { Multiple logistic regression } \\
95 \% \mathrm{Cl}\end{array}$ & $p$-value \\
\hline Baseline eGFR $\left(\mathrm{mL} / \mathrm{ml} / 1.73 \mathrm{~m}^{2}\right)$ & 0.89 & $0.85-0.92$ & $<0.001$ \\
\hline Baseline Haemoglobin (mg/dL) & 0.99 & $0.73-1.35$ & 0.95 \\
\hline Relative change of eGFR from baseline at time A (\%) & 0.98 & $0.98-1.01$ & 0.12 \\
\hline AKI 48 h p.o. no (ref.) vs. yes & 1.23 & $0.39-3.85$ & 0.72 \\
\hline Tumour diameter $(\mathrm{cm})$ & 0.93 & $0.71-1.21$ & 0.58 \\
\hline Tumour locus central (ref.) vs. peripheral & 1.35 & $0.56-3.15$ & 0.49 \\
\hline Surgical approach LNSS (ref.) vs. ONSS & 1.69 & $0.67-4.24$ & 0.26 \\
\hline Sex male (ref.) vs. female & 0.63 & $0.24-1.67$ & 0.35 \\
\hline Age (years) & 0.99 & $0.95-1.04$ & 0.75 \\
\hline $\mathrm{BMI}\left(\mathrm{kg} / \mathrm{m}^{2}\right)$ & 0.97 & $0.87-1.07$ & 0.50 \\
\hline Hypertension no (ref.) vs. yes & 1.62 & $0.66-4.00$ & 0.29 \\
\hline Ischaemia time (min) & 1.01 & $0.98-1.04$ & 0.55 \\
\hline Operative time (min) & 1.00 & $0.99-1.01$ & 0.86 \\
\hline Preoperative ureter stenting no (ref.) vs. yes & 1.26 & $0.41-3.86$ & 0.68 \\
\hline Intraoperative blood transfusions no (ref.) vs. yes & 0.95 & $0.08-11.05$ & 0.97 \\
\hline Postoperative complications no (ref.) vs. yes & 0.67 & $0.22-2.00$ & 0.47 \\
\hline Clavien-Dindo score $<3$ (ref.) vs. $\geq 3$ & 1.37 & $0.22-8.41$ & 0.73 \\
\hline
\end{tabular}

Multiple linear regression analysis for models 1 and 2 including ischaemia time as a continuous variable investigating predictors of the relative change (\%) of eGFR from baseline at (a) measurement time A (median, 1 day p.o.; IQR, 1-2) and at (b) at measurement time D (median, 13 months p.o.; IQR 12-15), and multiple logistic regression analysis for models 3 and 4 including ischaemia time as a continuous variable investigating (c) predictors for the development of postoperative AKI within $48 \mathrm{~h}$ p.o. and (d) predictors for the development of postoperative new-onset CKD stage $\geq 3$ (eGFR $<60 \mathrm{~mL} / \mathrm{min} / 1.73 \mathrm{~m} 2$ ) within measurement time $\mathrm{D}$ The regression models are based on pooled estimates from 100 imputed datasets. A $p$-value $<0.05$ is regarded as statistically significant eGFR estimated glomerular filtration rate, CKD chronic kidney disease, AKI acute kidney injury, LNSS laparoscopic nephron-sparing surgery, ONSS open nephronsparing surgery, $B M I$ body mass index, $O R$ odds ratio 